\title{
O exercício do Programa Saúde na Escola como prática da Equipe de Saúde Bucal na Atenção Básica: relato de experiência
}

The exercise of the School Health Program as a practice of Oral Health Team in Primary Care: report of an experience

El ejercicio del Programa Salud en la Escuela como práctica del Equipo de Salud Oral en Atención Primaria: reporte de una experiencia

André Rodrigo Justino da SILVA

Mestrando do Programa de Pós-Graduação em Odontologia pela Faculdade de Odontologia de Pernambuco, Universidade de Pernambuco (FOP/UPE), 54756-220 Camaragibe - PE, Brasil

\section{Resumo}

Introdução: O Programa Saúde na Escola se apresenta como uma forma concreta de promoção de saúde de extrema relevância para a assistência dos escolares facilitando o contato da população com os serviços odontológicos. Objetivo: Expor a realização de atividades do programa saúde na escola por uma equipe de saúde bucal do sistema único de saúde como uma experiência de saúde com escolares da região da Borborema do estado da Paraíba. Material e método: As ações envolveram 41 alunos de turmas de 05 e 12 anos de duas escolas municipais no município de Esperança- Paraíba. Foram realizadas visitas de reconhecimento dos locais para recolher todos os dados de cada instituição e planejamento das atividades. Como metodologias foram utilizadas jogo dos amigos e inimigos dos dentes; material educativo audiovisual sobre a técnica ideal de escovação; atividade de pintura com abordagem do tema dieta. Adicionalmente foi realizada aplicação tópica de flúor com Fluoreto de Sódio a $2 \%$ nas crianças com faixa etária de 05 anos. Relato de experiência: Os dois momentos de realização das atividades foram extremamente proveitosos e de uma participação ativa dos alunos que foram o público-alvo das ações que somada à cooperação da equipe de atenção básica resultou em êxito nos objetivos de promoção de saúde e de proteção específica. Conclusão: A realização de ações educativas no ambiente escolar através do programa saúde na escola é muito importante para melhorar a qualidade de vida da população.

Descritores: Promoção da Saúde; Serviços de Saúde Escolar; Saúde Bucal; Atenção Primária à Saúde; Sistema Único de Saúde.

\section{Abstract}

Introduction: The School Health Program presents itself as a concrete form of health promotion of extreme relevance for the assistance of students, facilitating the population's contact with dental services. Objective: To expose the accomplishment of activities of the school health program by an oral health team of the unified health system as a health experience with students from the region of Borborema in the state of Paraíba. Material and method: The actions involved 41 students from classes of 05 and 12 years old from two municipal schools in the city of Esperança- Paraíba. Site recognition visits were carried out to collect all data from each institution and activity planning. As methodologies were used game of friends and enemies of the teeth; audiovisual educational material on the ideal brushing technique; painting activity approaching the diet theme. Additionally, topical application of fluoride with $2 \%$ sodium fluoride was performed in children aged 5 years. Experience report: The two moments in which the activities were carried out were extremely fruitful and the students participated actively, as the target audience for the actions that, together with the cooperation of the primary care team, resulted in success in the objectives of health promotion and protection specific. Conclusion: The carrying out of educational actions in the school environment through the school health program is very important to improve the quality of life of the population.

Descriptors: Health Promotion; School Health Services; Oral Health; Primary Health Care; Unified Health System.

\section{Resumen}

Introducción: El Programa Salud en la Escuela se presenta como una forma concreta de promoción de la salud de extrema relevancia para la asistencia de los estudiantes, facilitando el contacto de la población con los servicios dentales. Objetivo: exponer la realización de actividades del programa de salud en la escuela por un equipo de salud bucal del sistema de salud unificado como una experiencia de salud con estudiantes de la región de Borborema en el estado de Paraíba. Material y método: En las acciones participaron 41 estudiantes de clases de 05 y 12 años de dos escuelas municipales del municipio de Esperança-Paraíba. Se realizaron visitas de reconocimiento del sitio para recopilar todos los datos de cada institución y planificación de actividades. Como metodologías se utilizaron juegos de amigos y enemigos de los dientes; material educativo audiovisual sobre la técnica de cepillado ideal; actividad de pintura que se acerca al tema de la dieta. Además, la aplicación tópica de fluoruro con fluoruro de sodio al $2 \%$ se realizó en niños de 5 años. Reporte de experiencia: Los dos momentos en los que se llevaron a cabo las actividades fueron extremadamente fructíferos y los estudiantes participaron activamente, como público objetivo de las acciones que, junto con la cooperación del equipo de atención primaria, dieron como resultado el éxito en los objetivos de promoción y protección de la salud. específico Conclusión: La realización de acciones educativas en el entorno escolar a través del programa de salud en la escuela es muy importante para mejorar la calidad de vida de la población.

Descriptores: Promoción de la Salud; Servicios de Salud Escolar; Salud Bucal; Atención Primaria de Salud; Sistema Único de Salud.

INTRODUÇÃO

O ambiente escolar há muito tempo se apresenta com múltiplas funções, entre elas a social que torna a escola um equipamento de desenvolvimento de ensino e aprendizagem associado às diversas formas de abrangência e envolvendo seu território adjacente. A promoção de saúde é uma dessas formas e se caracteriza por um estilo de vida, trabalho e aprendizagem que auxilie o desenvolvimento em saúde, tornando assim a escola uma instituição promotora de saúde. Desse modo, o Programa Saúde na Escola (PSE) se apresenta como uma forma concreta de promoção e deve ser planejado levando em conta o contexto escolar e social, além da percepção local da saúde dos alunos ${ }^{1}$.

O estabelecimento do PSE se deu a partir de um decreto presidencial e da união dos 
ministérios da educação e da saúde e se trata de uma política que delibera sobre ações de atenção aos estudantes da educação pública básica no país no âmbito escolar e/ou da Unidade Básica de Saúde (UBS) a partir da cooperação da Estratégia de Saúde da Família (ESF) que deve agir nas instituições de ensino presentes na sua área de abrangência, aproximando assim a equipe de saúde com a esfera da educação ${ }^{2}$.

Garantir a formação integral dos alunos da rede pública de ensino através de ações de prevenção e promoção à saúde foi o principal incentivo para estabelecimento do PSE, que possui múltiplos objetivos específicos, dentre eles promover saúde, prevenir agravos, estreitar o vínculo entre o Sistema Único de Saúde (SUS) e as redes de educação básica para facilitar o acesso dos alunos e de suas famílias, fortalecer o sistema de atenção social, facilitar a comunicação entre comunidade e profissionais de saúde e assegurar a participação comunitária nas políticas de atenção básica à saúde $^{2,3}$.

Assim observa-se a relevância do PSE para a assistência dos escolares como uma ferramenta de democratização de espaços e de acessos às estratégias de educação em saúde de modo geral e, especialmente no que se trata de saúde bucal, visto que facilitar cada vez mais - contato da população com os serviços odontológicos disponíveis no SUS em ambiente escolar pode auxiliar no fortalecimento da política preventiva como alternativa primordial de tratamento e, se tratando de crianças, adolescentes e jovens esse tipo de abordagem é de extrema importância para a conscientização o mais cedo possível da necessidade do cuidado com a saúde bucal ${ }^{4}$.

Desse modo, o objetivo desse estudo é expor a realização de atividades do programa saúde na escola por uma equipe de saúde bucal (ESB) do sistema único de saúde como uma experiência de saúde com escolares da região da Borborema do estado da Paraíba.

MATERIAL E MÉTODO

As ações foram realizadas no mês de outubro de 2019, com 41 alunos de turmas de 05 e 12 anos de duas escolas municipais no município de Esperança- Paraíba, pela equipe de saúde bucal componente da Estratégia de Saúde da Família (ESF) da unidade básica de saúde que geograficamente atendia os alunos das referidas escolas.

Inicialmente 0 cirurgião-dentista responsável realizou visitas de reconhecimento dos locais para, juntamente com seus respectivos diretores recolher todos os dados de cada instituição sobre número e faixa etária dos alunos, equipamentos disponíveis para utilização nas ações e planejamento das mesmas, levando também em conta os anseios verificados com os gestores de cada escola.

Após planejamento minucioso com toda a equipe componente da ESF foi solicitada ajuda dos Agentes Comunitários de Saúde (ACS) para suporte nos dias designados para a realização das atividades propostas. Como metodologias para viabilizar as ações foram utilizadas: jogo dos amigos e inimigos dos dentes; material educativo audiovisual sobre a técnica ideal de escovação; atividade de pintura com abordagem do tema dieta. Adicionalmente foi realizada aplicação tópica de flúor com Fluoreto de Sódio a $2 \%$ nas crianças com faixa etária de 05 anos.

RELATO DE EXPERIÊNCIA

Realizar a visita prévia às escolas e conversar com seus diretores para se adequar à rotina deles foi essencial para o sucesso das atividades realizadas. Além disso, contar com o auxílio dos agentes comunitários de saúde se mostrou de extrema importância tanto por ser um recurso humano para ajuda na realização de cada etapa quanto por conhecer muito bem os alunos participantes, podendo assim repassar qualquer informação para as famílias deles mais facilmente.

A primeira faixa etária abordada foi de 12 anos, com uma turma de 26 alunos (Figura 1) com os quais foi realizada inicialmente a dinâmica de colorir que consistia na entrega de um conjunto de ilustrações de diversos alimentos (maçã, laranja, chocolate, pirulitos, leite, bombom e água) para que eles pudessem pintar todos e utilizá-los na dinâmica seguinte que foi 0 jogo dos amigos e inimigos dos dentes, no qual era discutido se cada gravura colorida consistia em um alimento "amigo do dente" ou "inimigo do dente", ou seja, se fazia bem à saúde geral e à integridade do dente.

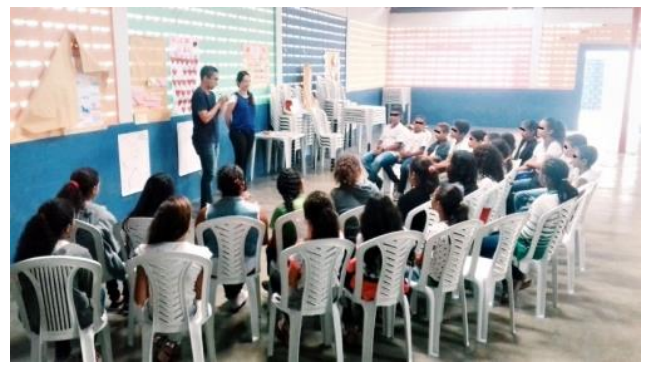

Figura 1: Atividade com alunos de 12 anos de idade.

Desse modo, cada aluno colava sua pintura em um dos dois dentes gigantes feitos em cartolina e colados na parede (dente feliz e dente triste) e era trabalhado assim o tema dieta com uma abordagem lúdica e interativa, com 
participação ativa dos alunos. Em seguida já foi trabalhado 0 tema escovação com a demonstração da técnica ideal com o auxílio de macromodelo e escova através de linguagem simples e de fácil percepção. Ainda nesse momento alguns alunos foram convidados para demonstrar no modelo e dessa forma exercer e reforçar o que tinha acabado de ser passado para eles (Figura 2). Por fim foi estimulado nesses alunos que eles repassassem o que foi aprendido nas atividades para outras pessoas, especialmente no ambiente familiar, desse modo agindo como agentes multiplicadores das informações de promoção de saúde bucal.

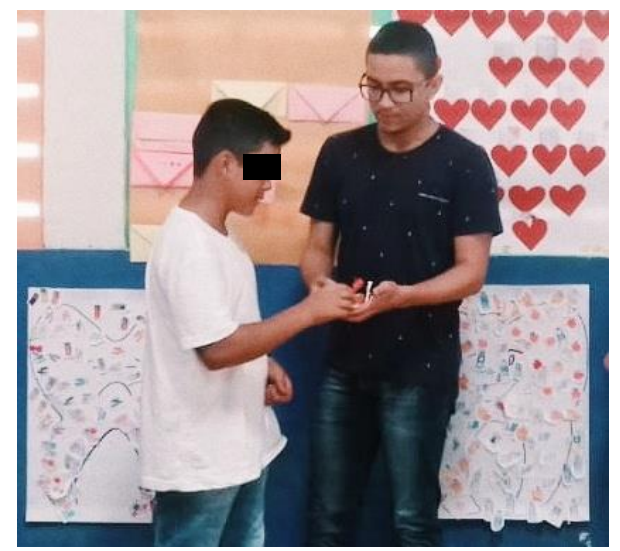

Figura 2: Reforço da técnica de escovação dentária em macromodelo.

Em outro momento foi realizada a atividade com a turma de 15 alunos com a faixa etária de 05 anos, que ocorreu com o uso de material audiovisual na forma de vídeo educativo adequado para a idade projetado na sala de aula com música de instrução à técnica adequada de escovação (Figura 3). Após esse momento foi feita atividade de pintura seguida do jogo dos amigos e inimigos do dente da mesma forma que já foi descrita.

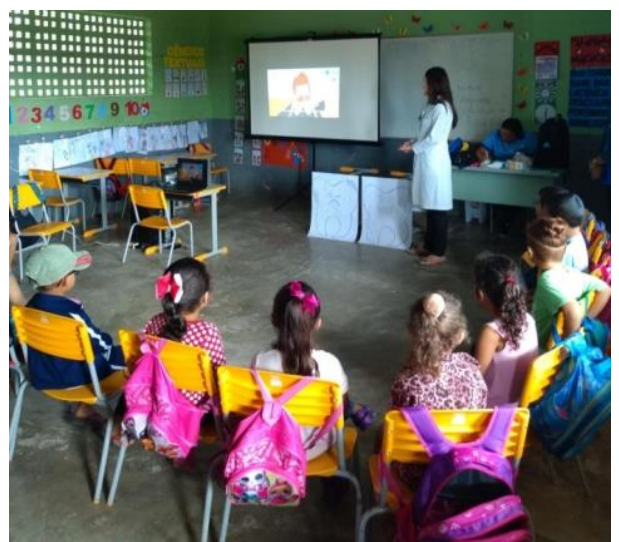

Figura 3: Apresentação do material audiovisual.

Ao fim da parte educativa foi realizada a aplicação tópica de flúor em todos os alunos dessa turma com o uso de flúor neutro a $2 \%$ de aplicação tópica aplicado em um rolete de algodão para ser levado em contato com os dentes (Figura 4). Ao final das aplicações os alunos foram instruídos que não poderiam comer nem beber nada no intervalo de 30 minutos e as professoras ficaram responsáveis por esse controle.

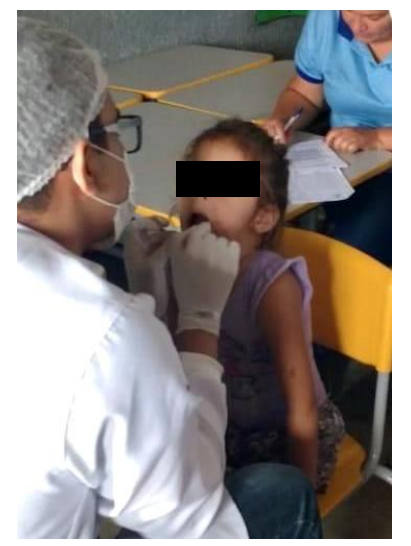

Figura 4: Aplicação tópica de flúor.

Assim observou-se uma participação ativa dos alunos que foram o público-alvo das ações que somada à cooperação da equipe de atenção básica resultou em êxito nos objetivos de promoção de saúde e de proteção específica, de modo a colaborar na efetivação das funções assistenciais da equipe de saúde bucal responsável.

DISCUSSÃO

A presença do cirurgião-dentista como instrumento constante de promoção de saúde no programa saúde na escola é muito importante, já que se trata de um profissional com muitos artifícios para tal função. O resultado dessa participação é a melhoria da qualidade de vida do público-alvo das atividades ${ }^{5,6}$.

De acordo com Sousa et al. ${ }^{5}$, uma vez que a escola é o ambiente de desenvolvimento intelectual, de hábitos, valores e costumes para a faixa etária de crianças e adolescentes, utilizar - ambiente escolar para propagação de medidas preventivas e de promoção se mostra bastante adequado. O grupo de escolares, especialmente o grupo com menores idades, possui grande potencial de imitar as ações que observam, sendo assim mais passíveis de correção de hábitos e condutas inadequadas. Portanto é necessária a ampliação da abrangência do PSE para torná-lo mais popular e, assim considerar todas as faixas etárias presentes no ambiente escolar.

Em uma pesquisa feita por Moura et al. ${ }^{7}$ com professores de escolas públicas feito no estado de Paraná, 98,1\% deles afirmaram que abordam temas relevantes à saúde bucal nas suas aulas e que consideram um tema extremamente relevante. Porém, ao aprofundar o olhar observou-se que o conhecimento de alguns temas específicos ainda não está 
completamente claro, principalmente sobre traumatismo dentário, já que todos os professores avaliados relataram que nunca receberam orientação ou treinamento da conduta adequada frente a algum caso.

Portanto, como posto por Oliveira et al. ${ }^{8}$, as ações de promoção de saúde bucal nas escolas devem focar também nos professores que são instrumentos muito importantes de propagação e reforço, no cotidiano da sala de aula, das informações repassadas. Para isso fornecer subsídios teórico-práticos para esses profissionais se faz necessário como prática das ESB. A realização de ações de capacitação dos educadores, sobre temas identificados como deficiências a partir de escuta prévia, pelos profissionais da unidade básica de saúde se mostra uma sugestão muito pertinente para criação de agentes multiplicadores de saúde bucal.

Assim como verificado na descrição do presente estudo, Rodrigues et al. ${ }^{9}$ afirmam que 0 uso de metodologias ativas e 0 desenvolvimento de projetos integradores aperfeiçoam 0 aprendizado dos alunos nas práticas do programa saúde na escola e despertam 0 interesse pelas práticas mais saudáveis. Em um estudo de Oliveira et al. ${ }^{10}$, que colheu a avaliação por parte de pais, professores e enfermeiros da situação das ações de saúde bucal no PSE verificou-se uma concepção como insuficiente 0 grau de participação da equipe de saúde bucal, porém com grande ressalva à falta de apoio do governo com incentivo e material que propicie uma parceria entre o ambiente escolar e as entidades de saúde.

Ainda nesse estudo observou-se que os professores e os enfermeiros por muitas vezes se vêem, durante realização de atividades em ambiente escolar, como os responsáveis por identificar alterações bucais nos escolares, sem possuírem nenhuma formação acadêmica para isso. Portanto, conforme as percepções de Mendes et al. ${ }^{11}$ é necessária articulação sólida entre toda a equipe de saúde da família, incluindo o cirurgião-dentista para que nenhuma alteração passe despercebida. Por outro lado, se faz essencial a realização, por parte do profissional da odontologia de educação permanente com seus colegas de equipe, para de alguma forma capacitá-los como agentes de melhoria da qualidade de saúde bucal dos escolares, bem como dos pacientes em geral.

Ao avaliar a percepção de professores de educação infantil sobre a aplicabilidade de ações de educação em saúde bucal, Rodrigues et al. ${ }^{12}$ observaram a consciência por parte deles da importância dessas ações e de suas repercussões positivas no organismo. De modo mais específico foi aferido que os educadores afirmam que as abordagens da forma correta de higienização e de uma alimentação saudável se caracterizam como combinação essencial para aquisição de uma boa saúde bucal de préescolares e de alunos de ensino fundamental.

CONCLUSÃO

De modo geral conclui-se que a realização de ações educativas no ambiente escolar através do programa saúde na escola é muito importante como forma de melhorar a qualidade de vida da população, exercer a odontologia social e preventiva e aproximar a esfera educacional à saúde, tal qual aconteceu nas atividades relatadas no presente estudo. Além disso, é importante saber que, assim como na experiência descrita neste artigo, a promoção de saúde na escola possui dificuldades de execução e falta de incentivo, sendo necessária tanto a atenção maior do governo quanto a articulação de toda a estratégia de saúde da família, para assim obter uma abordagem holística dos escolares da educação pública brasileira.

REFERÊNCIAS

1. Graciano AMC, Cardoso NMM, Mattos FF, Gomes VE, Borges-Oliveira AC. Promoção da Saúde na Escola: história e perspectivas. J Health Biol Sci. 2015;3(1):34-8.

2. Brasil. Presidência da República. Poder Executivo. Decreto no 6.286, de 5 de dezembro de 2007. Institui o Programa Saúde na Escola PSE, e dá outras providências. Diário Oficial da União 2007; 5 dez.

3. Brasil. Ministério da Saúde (MS). Ministério da Educação. Orientações sobre o programa saúde na escola para a elaboração dos projetos locais. 2008; Brasília: MS.

4. Ferreira IRC, Vosgerau DSR, Moysés SJ, Moysés ST. Diplomas Normativos do Programa Saúde na Escola: análise de conteúdo associada à ferramenta ATLAS TI. Ciên Saúde Colet. 2012;17(12):3385-3398.

5. Sousa JB, Pinheiro Neto MB, Lobo VFB, Carneiro SV, Silva CHF. Benefícios da inserção do cirurgião dentista no programa saúde na escola. Jornada odontológica dos acadêmicos da católica. JOAC. 2016;2(2).

6. Bezerra BX, Fernandes ME, Henschel FAN, Molina LM, Nakaie DH, Petyk WS et al. Medidas preventivas e curativas em saúde bucal nos municípios de Mandaguari e de Sarandi em conjunto com a Pastoral da Criança: um relato de experiência. Arch Health Invest. 2020;9(4):367-70.

7. Moura AMG, Silva ROC, Zermiani TC, Ditterich RG. Conhecimento sobre saúde bucal de 
professores de escolas públicas em ColomboPR. Rev Espaço para a Saúde. 2018;19(1): 57-64.

8. Oliveira EL, Riatto SG, Vieira APSB, Carvalho G, Fonseca M, Guedes V et al. Importância do nível de conhecimento dos professores de escola pública do ensino fundamental sobre saúde bucal: revisão de literatura. Rev Campo do Saber. 2018;4(5):2-16.

9. Rodrigues CAL, Sá-Silva JR, Rocha AHSG. Conhecimentos e práticas em saúde bucal na escola: relato de experiências. REAMEC. 2020; 8(1):404-17.

10. Oliveira EEG, Arantes DC, Nascimento LS, Pontes FSC. Oral health assessment in school program health: who and how? RGO Rev Gaúch Odontol. 2018;66(2):154-59.

11. Mendes JDR, Freitas CAS, Dias MSA, Fernandes DR. Análise das atividades de educação em saúde realizadas pelas equipes de saúde bucal. Rev Bras Promoção da Saúde. 2017;30(1):13-21.

12. Rodrigues JC, Sousa TBP, Joaquim DC, Benedito FCS, Cruz GS, Leite ACRM. Percepção de professores quanto à aplicabilidade das ações de educação em saúde bucal. Rev Diálogos Acadêmicos. 2016; 5(2):86-91.

\section{CONFLITO DE INTERESSES}

Os autores declaram não haver conflitos de interesse

AUTOR PARA CORRESPONDÊNCIA

\section{André Rodrigo Justino da Silva}

Rua São José, 429, Bairro Santo Antônio.

58701-120 Patos PB, Brasil

E- mail: a.rodrigo2010@hotmail.com

Submetido em 06/04/2020

Aceito em 23/10/2020 